\title{
A doubly functionalized mesoporous silica nanoscavenger for the analytical extraction of triphenyltin from water
}

\author{
Awad Aqeel Al-rashdi ${ }^{1,2}$ \\ ${ }^{1}$ Department of Chemistry, Al-Qunfudah, Saudi Arabia \\ ${ }^{2}$ Umm Al-Qura University, Al-Qunfudah University College, Al-Qunfudah, Saudi Arabia
}

Email address:

aarashdi@uqu.edu.sa

\section{To cite this article:}

Awad Aqeel Al-rashdi. A Doubly Functionalized Mesoporous Silica Nanoscavenger for the Analytical Extraction of Triphenyltin from Water. Science Journal of Analytical Chemistry. Vol. 1, No. 1, 2013, pp. 1-6. doi: 10.11648/j.sjac.20130101.11

\begin{abstract}
A new analytical procedure has been developed for the determination of triphenyltin(TPT), diphenyltin (DPT), and monophenyltin (MPT) in water samples. The method is based on the use of the dual functionalization of mesoporous silica with diol and $\mathrm{C}_{18}$ alkane groups for the collection of TPT and its derivatives, DPT and MPT, from water samples, followed by hexylation of the target matrices using the Grignard reagent and quantification by gas chromatography with pulsed flame photometric detection. The nanoscavenger concept replaces conventional solid- and liquid-phase extractants with nanosized particles that can be readily dispersed in aqueous samples. Analyte partitioning between the solid and liquid phases occurs as the solid moves through the sample as a colloidal sol. By tailoring the size of the particles to approximately $250 \mathrm{~nm}$ in diameter, they can be readily recovered together with the analytes by simple filtration or centrifugation. Recoveries of TPT, DPT, and MPT chloride spiked matrices ranging from $89.3 \pm 2.1$ to $97.1 \pm 1.3$ in seawater samples ( $n=9$ samples). The limit of detection obtained was typically in the range of $0.5-5 \mathrm{ng} \mathrm{Sn} / \mathrm{L}$. The proposed method shows excellent linearity in the range of $0.5-2 \mathrm{ng} \mathrm{Sn} / \mathrm{L}$ and good repeatability (RSD $\leq 5 \%$ at $0.02 \mathrm{ng}$ TPT (as $\mathrm{Sn}) / \mathrm{L}$ ). The method performance is demonstrated with real seawater samples.
\end{abstract}

Keywords: Triphenyltin, Mesoporous Silica, Nanoscavenger, Colloidal Sol, Seawater Sample

\section{Introduction}

Organotin compounds (OTC) have been extensively used in many human activities. Triphenyltin (TPT) and tributyltin (TBT) are mainly used as a biocide, especially in antifouling paints [1]. TPT compounds have also been widely used in agriculture as fungicides [2].TPT compounds are a group of organotins conforming to a general formula $\left(\mathrm{C}_{6} \mathrm{H}_{5}\right)_{3} \mathrm{SnX}$, where $\mathrm{X}$ is an anion or anionic group, such as chloride, hydroxide, and acetate [3]. Various studies have shown that TPT and its degradation products caused extremely harmful effects to certain marine gastropods even at low ng/L levels [4]. As a result, the European Commission banned the use of TPT-containing antifouling paints on the ships, and it was included in the list of priority pollutants in the field of water policy in the EU Water Framework Directive [5].

The analytical method of organotin determination typically involves the following steps: extraction from the sample matrix, derivatization, chromatographic separation, and selective detection. Liquid chromatography (LC) and gas chromatography (GC) coupled with an elemental selective detector, such as atomic absorption spectrometry (AAS) [6,7], microwave-induced plasma atomic emission spectrometry (MIP-AES) [8,9], inductively coupled plasma mass spectrometry (ICP-MS) [10], flame photometric detection (FPD) [11], and, more recently, pulsed flame photometric detection (PFPD) have been commonly used [12]. For derivatization, alkylation with Grignard reagents and ethylation with sodium tetraethylborate are used $[13,14]$.

Extraction of OTC from environmental samples is the most difficult part of an OTC speciation analysis because of the limited stability of the analyte and the strong association of the analyte and the matrices. Moreover, the choice of the extraction technique is very important because TPT may convert to DPT or MPT if exposed to very hard extraction conditions. On the other hand, the extraction will not be complete if the conditions are too 
weak [15].

Different extraction and preconcentration techniques have been used for extraction of OTC from environmental samples. Commonly used extraction techniques include solvent extraction [16], solid-phase extraction [17], and solid-phase microextraction (SPME) [18]. However, there are some difficulties associated with the use of solvent extraction. It requires large amounts of hazardous solvents and is time-consuming. Also, it suffers from low OTC recovery.

The most recently developed method for the extraction and preconcentration of analytes from an aqueous solution is solid-phase dispersion (SPDE) [19]. This method introduces a novel concept for the extraction and preconcentration of materials from an aqueous solution. Extraction is performed based on the dispersion of submicron silicas (nanoscavengers) in an aqueous solution. The nanoscavenger particles move naturally through the samples and scavenge the analytes. Continuous physical movement is therefore not required. The analyte-loaded nanoscavenger can then be recovered from the sample by filtration [20].

\section{Experimental}

\subsection{Materials}

Deionized water $\left(5 \mathrm{M} \Omega-\mathrm{cm}\right.$ at $\left.25^{\circ} \mathrm{C}\right)$ was produced using an ELGA water purifier with hexane (95\% HPLC grade). $n$ Toluene (99.99\%, HPLC grade) was purchased from Fisher (Loughborough, UK),(dried by fractional distillation under $\mathrm{N}_{2}$ from over phosphorus $(\mathrm{V})$ oxide $\mathrm{P}_{2} \mathrm{O}_{5}$, hexylmagnesium bromide $(2.0 \mathrm{M}$ solution in diethylether), triphenyltin chloride (TPTCl, 96\%), diphenyltin dichloride (DPTCl, 96\%), monophenyltin trichloride (MPTCl, 95\%) and tetrapentyltin (TPeT, 97\%) were obtained from Aldrich (Gillingham, UK). Ethylene glycol(analytical grade),sodium hydroxide (laboratory grade), and hydrochloric acid (37\%, laboratory grade) were purchased from Fisher (Loughborough, UK). Tetramethoxysilane(98\%),3-glycidoxypropyltrimethoxysilane (98\%), and octadecyltrichlorosilane (+90\%) were obtained from Aldrich (Gillingham, UK).

(1-Dodecyl)trimethylammonium bromide (99\%) was purchased from Alfa Aesar (Lancashire, UK). Polyvinylidene fluoride (PVDF) membrane filters (diameter of $47 \mathrm{~mm}$, pore sizes of $0.22 \mu \mathrm{m}$ ) were supplied by Millipore (Billerica, USA).

\subsection{Apparatus}

For the analysis of TPT compounds, a Varian 3800 gas chromatograph equipped with a pulsed flame photometric detection PFPD (GC-PFPD) and a Varian 1079 temperature programmable split/splitless injector were used. The GC separation was carried out using a capillary column. The operating conditions used for the gas chromatographic detection of phenyltin are shown in Table1.
Table1. Operating Conditions of GC-PFPD

\begin{tabular}{ll}
\hline Parameters & Conditions \\
\hline Column & $\begin{array}{l}\text { Sigma-Aldrich, SA-1 type, fused } \\
\text { silica }(30 \mathrm{~m} \times 0.25 \mathrm{~mm} \times 0.25 \mu \mathrm{m})\end{array}$ \\
Nitrogen \\
Injector temperature program & $130^{\circ} \mathrm{C}(1 \mathrm{~min})$ to $270^{\circ} \mathrm{C}$ at $40^{\circ} \mathrm{C} / \mathrm{min}$ \\
& $50^{\circ} \mathrm{C}(3 \mathrm{~min})$ to $120^{\circ} \mathrm{C}$ at $50^{\circ} \mathrm{C} / \mathrm{min}$ to \\
& $120^{\circ} \mathrm{C}$ at $7^{\circ} \mathrm{C} / \mathrm{min}$ to \\
Oven program & $280^{\circ} \mathrm{C}$ at $20^{\circ} \mathrm{C} / \mathrm{min}$, held for $13 \mathrm{~min}$ \\
& $1.00 \mathrm{~mL} / \mathrm{min}$ \\
\hline
\end{tabular}

\subsection{Standard Solutions of Organotins}

Standard stock solutions of individual organotin chlorides (TPTCl, DPTCl, MPTCl) $(500 \mathrm{mg} / \mathrm{L}$ as Sn) were prepared by dissolving organotin chloride compounds in hexane individually and stored in the dark at $4^{\circ} \mathrm{C}$. The mixed working solutions of TPTCl, DPTCl, and MPTCl ranging from 0.5 to $2 \mathrm{ng} \mathrm{Sn} / \mathrm{L}$ were prepared daily from the stock solutions.

\subsection{Synthesis of Octadecyl-diol Silica Nanoscavenger Particles}

Octadecyl-diol silica particles have been synthesized and described previously by Mohammed Algaradah [21]. Briefly, The synthesis of nanoscavenger silica was carried out using dodecyltrimethylammonium bromide as a template and tetramethoxysilane as a silica precursor. The silica product was modified with 3-glycidoxypropyl groups, and the 3-glycidoxypropyl-nanoscavenger silica was reacted with trichloro(octadecyl)silane. The epoxy ring of the octadecyl-modified silica was opened to gain octadecyldiol-modified silica.

\subsection{Extraction and Preconcentration of Phenyltins Using Octadecyl-diol Silica Nanoscavenger}

$200 \mathrm{mg}$ of octadecyl-diol-modified silica was sonicated in $10 \mathrm{~mL}$ of deionized water. The suspension was transferred to a $1000 \mathrm{~mL}$ volumetric flask, which was then filled to the mark with deionized water. $5 \mu \mathrm{l}$ of phenyltin mixed standard $(0.3,0.2,0.7 \mathrm{ng} \mathrm{Sn} / \mathrm{L}$ of TPTCl, DPTCl, and $\mathrm{MPTCl}$, respectively) was added to the flask using a Hamilton Microliter syringe.

The flask was left for $4 \mathrm{hr}$. The suspension was then vacuum filtered using a $0.22 \mu \mathrm{m}$ polyvinylidene fluoride (PVDF) polymer membrane filter. The filter paper was carefully transferred to a $20 \mathrm{~mL}$ glass vial and dried overnight in a desiccator using an activated silica gel desiccant.

The dried filter paper was carefully cut into small pieces inside the polytetrafluoroethylene (PTFE) glass vial. $2 \mathrm{~mL}$ of hexane was added into the glass vial and mixed well with the filter paper pieces. $1 \mathrm{~mL}$ of hexylmagnesium bromide ( $2 \mathrm{M}$ in ether) was added and shaken violently. The reaction was left to proceed at ca. $20^{\circ} \mathrm{C}$ for $2 \mathrm{hr} .2 \mathrm{~mL}$ of hydrochloric acid solution $(5 \% \mathrm{v} / \mathrm{v})$ was added to quench 
the excess of the Grignard reagent. The glass vial was shaken well to ensure the complete deactivation of the reagent. Then the aqueous layer was discarded, and the hexane layer was dried with activated $\mathrm{Na}_{2} \mathrm{SO}_{4}$.

The dried hexane layer was transferred to a $2 \mathrm{~mL}$ volumetric flask, and the flask was filled to the mark using hexane. $5 \mu \mathrm{L}$ of the solution was injected into a gas chromatograph, which was fitted with a capillary column, coupled with a pulsed flame photometric detector (GCPFPD).

\subsection{Quantification}

The hexylated organotin standards were prepared by hexylation of the appropriate organotin salts with an excess of Grignard reagent, following the derivatization procedure described above.

Quantitative analysis was based on the calibration curves obtained by the analysis of hexylated OTC in the range of 0.5-2 ng Sn/L. Linear calibration graphs were constructed by least-squares regression of concentration versus peak height. Good linearity was found in the concentration range tested (0.5-2 ng $\mathrm{Sn} / \mathrm{L})$, with determination coefficients higher than 0.98 in all cases.

Tetrapentyltin (TPeT) was suitable for use as the internal standard (IS) for the quantitative calculation of other organotin species because of its absence in environmental samples. $2 \mathrm{ng} \mathrm{Sn} / \mathrm{L}$ of the TPeT solution was used to correct the concentration values of analytes for losses during manipulation, extraction inefficiency, incomplete conversion during derivatization, and evaporative losses. The identification of the OTC was based on the retention time (defined as the retention time averages \pm 3 standard deviations of the retention times).

\section{Results and Discussion}

\subsection{Characterizations of the Octadecyl-diol Silica Nanoscavenger Particles}

The thermogravimetric analysis in Figure1(A) shows a weight loss of $6 \%$ between $270^{\circ} \mathrm{C}$ and $350^{\circ} \mathrm{C}$ resulting from the highly exothermic composition of the 3 (glycidoxy)propyl group. Figure1(B) shows a weight loss of $15 \%$ between ca. $270^{\circ} \mathrm{C}$ and $430{ }^{\circ} \mathrm{C}$ resulting from a controlled composition of the $\mathrm{C}_{18}$ group.

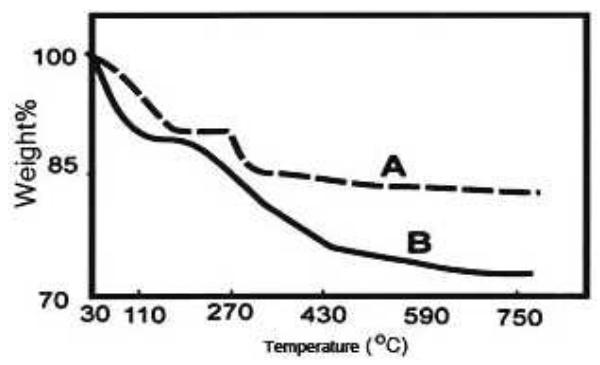

Figure1. Thermal Analysis of Modified Silica (in air):(A) 3-glycidoxypropyl silica,(B) $C_{18} /$ diol-modified silica
The Fourier transform infrared spectroscopy (FTIR) of the mesoporous silica before and after modification was carried out. The spectra in Figure 2 shows bands for asymmetric and symmetric stretching for $\mathrm{C}-\mathrm{H}$ from $-\mathrm{CH}_{2}$ and $-\mathrm{CH}_{3}$ groups located at $2800-3000 \mathrm{~cm}^{-1}$. The band at $1468 \mathrm{~cm}^{-1}$ is attributed to $\mathrm{C}-\mathrm{H}$ bending. The results are The results are closely in agreement with published data [22,23] and indicate that the octadecyl group is chemically bonded on the surface of the silica.

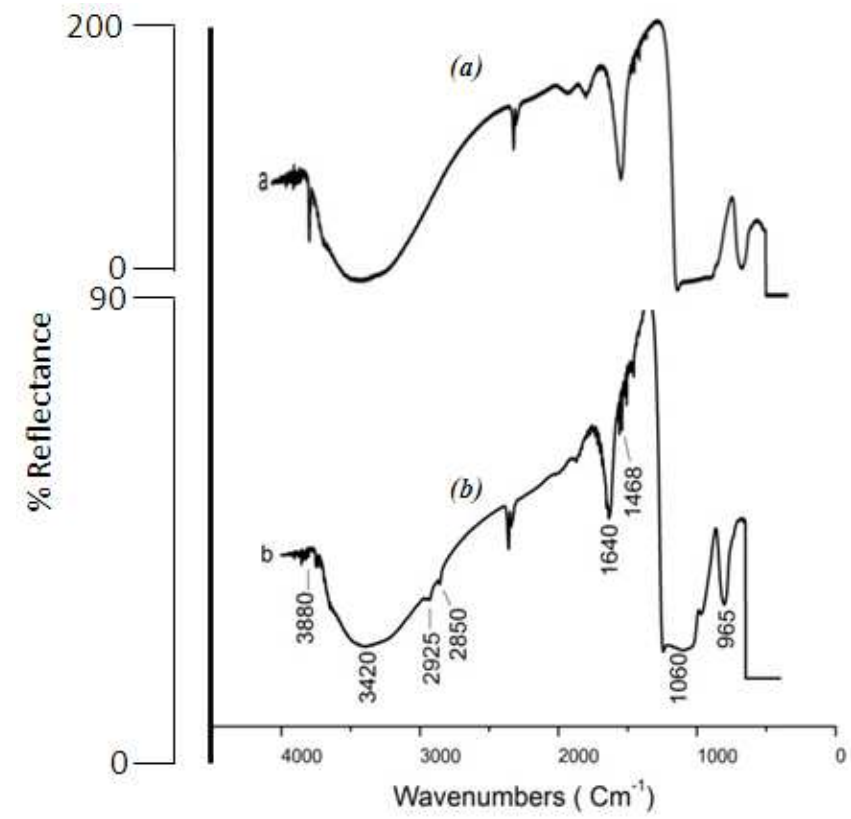

Figure2. Fourier Transform Infrared Spectroscopy of (a) Mesoporous Silica (Before Modification) and (b) the HOC 18 -nanoscavenger

The scanning electron microscopy (SEM) image of the $\mathrm{C}_{18}$ /diol-modified silica in Figure 3 shows that the material is spherically shaped with a size approximately in the range of 200-290 nm with a mean diameter of $250 \pm 110 \mathrm{~nm}$ based on the measurement of some individual particles using the SEM measurement tools. The particles are clearly visible and separated from each other. However, some aggregated particles were observed. This is due to Van der Walls and electrostatic interaction of alkyl chains grown on the surface of the particles.

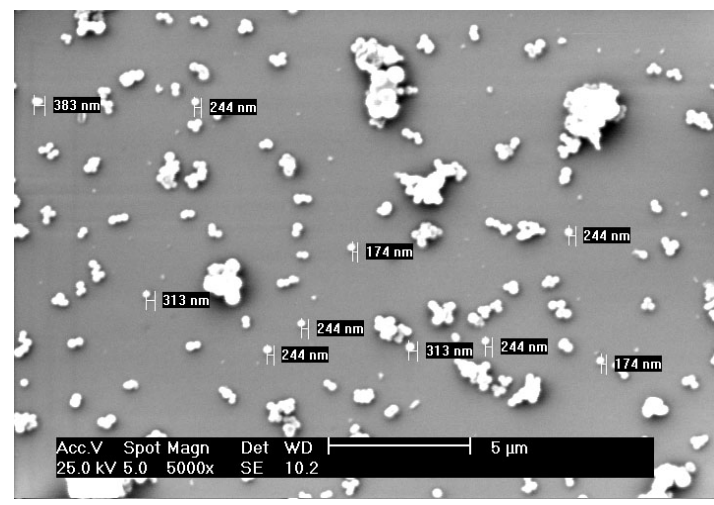

Figure3. SEM Image of the $H_{18} C_{18}$-nanoscavenger 
The nitrogen adsorption-desorption isotherm of $\mathrm{C}_{18} /$ diolmodified silica in Figure4 shows a typical type of isotherm with an $\mathrm{H} 1$ hysteresis loop as defined by International Union of Pure and Applied Chemistry (IUPAC). The Brunauer-Emmett-Teller (BET) surface area, calculated using the multipoint method, of the $\mathrm{C}_{18}$ /diol-modified silica was very high (approximately $1510 \mathrm{~m}^{2} / \mathrm{g}$ ). The position of sharp inflection in the $\mathrm{P} / \mathrm{P}^{0}$ range from 0.6 to 0.8 of the isotherm is clearly related to a diameter in the mesopore range [24, 25].

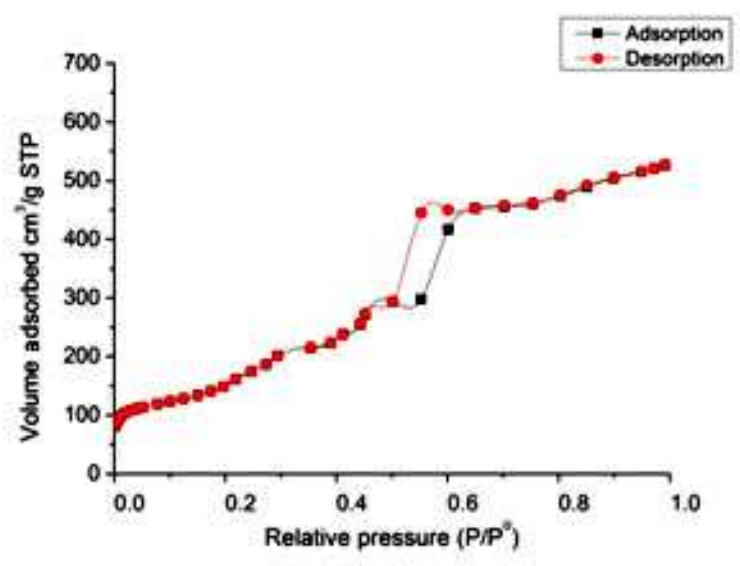

Figure4. Nitrogen Adsorption-Desorption Isotherm of the Unmodified Mesoporous Silica and the $\mathrm{HOC}_{18}$-nanoscavenger

\subsection{Rate of Phenyltin Chloride Uptake by Octadecyl-diol-Silica Nanoscavenger}

Experiments were carried out to assess the time required to achieve the quantitative collection of phenyltin chlorides from an aqueous solution by nanoscavenger dispersion. Octadecyl-diol-modified silica was dispersed in a spiked sample solution for time periods ranging from $30 \mathrm{~min}$ to 4 hr. The analysis of the TPT, DPT and MPT recovered from the nanoscavenger in Figure5 shows that maximum quantitative recovery was achieved within a $4 \mathrm{hr}$ equilibration period.

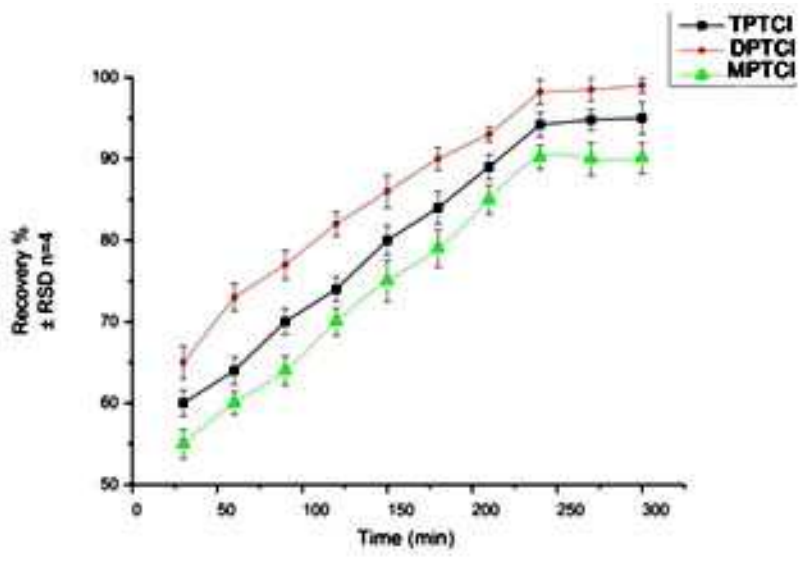

Figure5. Rate of Phenyltin Chlorides Collected by An octadecyl-diol Nanoscavenger

\subsection{Derivatization Reaction Time}

The rate of the Grignard reagent with the collected phenyltinchloride-loaded nanoscavenger was assessed. Recoveries of phenyltin compounds were gradually increased by increasing the reaction time up to $120 \mathrm{~min}$, but no significant improvement was obtained after that time. Recoveries improved from $60 \pm 1.6$ to $96.6 \pm 3.6,64 \pm 2.1$ to $98.1 \pm 1.7$, and $56 \pm 1.8$ to $90.1 \pm 1.9$ for TPT, DPT, and MPT, respectively. The reasons for this are: some phenyltin chloride may be localized within the pores of the nanoscavenger and some of the nanoscavenger particles are held inside the pores of the filter paper. Therefore, the Grignard reagent needs time to penetrate the pores to react with all the phenyltin chloride. In addition, some of the Grignard may destroyed by localized wet regions in the silica.

\subsection{Performance Data}

\subsubsection{Detection Limit}

The limit of detection was assessed as the lowest phenyltin concentration injected that yielded a signal of 3 times the noise from a blank. A blank was measured 4 times per day over 4 days, yielding an average noise of $300 \pm 120$ $\mu \mathrm{V}$. The detection limit of TPT, DPT, and MPT were 0.5 , 1.0 , and $3.5 \mathrm{ng} \mathrm{Sn} / \mathrm{L}$ at $95 \%$ confidence, respectively.

\subsubsection{Reproducibility and Repeatability}

By using the analytical procedure described above, repeatability for TPTs was determined by the analysis of the mixed standard solution of TPTCl, DPTCl, and MPTCl at $0.05 \mathrm{ng} \mathrm{Sn} / \mathrm{L}$, done 6 times consecutively and expressed as relative standard deviation (RSD\%). Values lower than $5 \%$ were obtained for all compounds. The reproducibility for TPTs was assessed by the analysis of the mixed standard solution of TPTCl, DPTCl, and MPTCl at $0.05 \mathrm{ng}$ $\mathrm{Sn} / \mathrm{L}$ on 3 different days (6 times each day) and expressed as $\mathrm{RSD} \%$. Values lower than $8 \%$ were obtained for all compounds.

\subsection{Extraction Efficiency}

The effectiveness of the dispersion extraction and preconcentration approach with octadecyl-diol silica nanoscavengers was assessed for TPT, DPT, and MPT. Recovery for all compounds at a low concentration level was performed in order to assess the extraction efficiency of the proposed method. For this purpose, 9 seawater samples were spiked with phenyltin chlorides and processed as described above. Recoveries of TPT, DPT, and MPT chloride spiked matrices ranging from $91.3 \pm 3.2$ to $98.1 \pm 1.7$ in seawater samples at concentrations of $1.5,1$, and $3.5 \mathrm{ng} \mathrm{Sn} / \mathrm{L}$ of TPT, DPT, and MPT, respectively. Figure6 shows a typical chromatogram obtained from a spiked water sample extract using the octadecyl-diol silica nanoscavenger. 


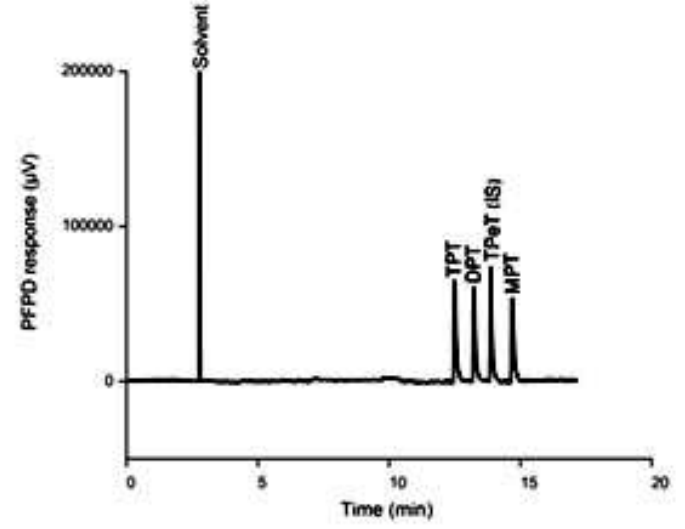

Figure6. Typical Chromatogram of Hexylated Phenyltins Derived from Spiked Deionized Water Samples (Concentrations of All Phenyltins were $1.25 \mathrm{ng} \mathrm{Sn/L)}$

\subsection{Application to Water Samples}

To assess the applicability of the developed method, 9 seawater samples were analyzed. Samples were collected from different areas of Al-Qunfudah Beach (south of Saudi Arabia) in June 2012 (see Figure7). Samples were collected in plastic bottles and analyzed $(n=3)$ without filtration. Quality assurance of the results was established by analyzing a blank extract and a calibration curve in each compound (TPT, DPT, and MPT) at the same time as the analysis of the set of samples. The TPeT solution was used as the IS to correct the variability of the results. TPT concentrations ranged from 2.6 to $8.1 \mathrm{ng} \mathrm{Sn} / \mathrm{L}$, with an average concentration of $4.9 \pm 1.1 \mathrm{ng} \mathrm{Sn} / \mathrm{L}$; DPT concentrations from 1.7 to $6.2 \mathrm{ng} \mathrm{Sn} / \mathrm{L}$, with an average concentration of $3.4 \pm 0.6 \mathrm{ng} \mathrm{Sn} / \mathrm{L}$; and MPT concentrations from 1.1 to $4.2 \mathrm{ng} \mathrm{Sn} / \mathrm{L}$, with an average concentration of $2.6 \pm 0.5 \mathrm{ng} \mathrm{Sn} / \mathrm{L}$. All these results are understandable since both commercial vessels and pleasure yachts are regularly used in this area.

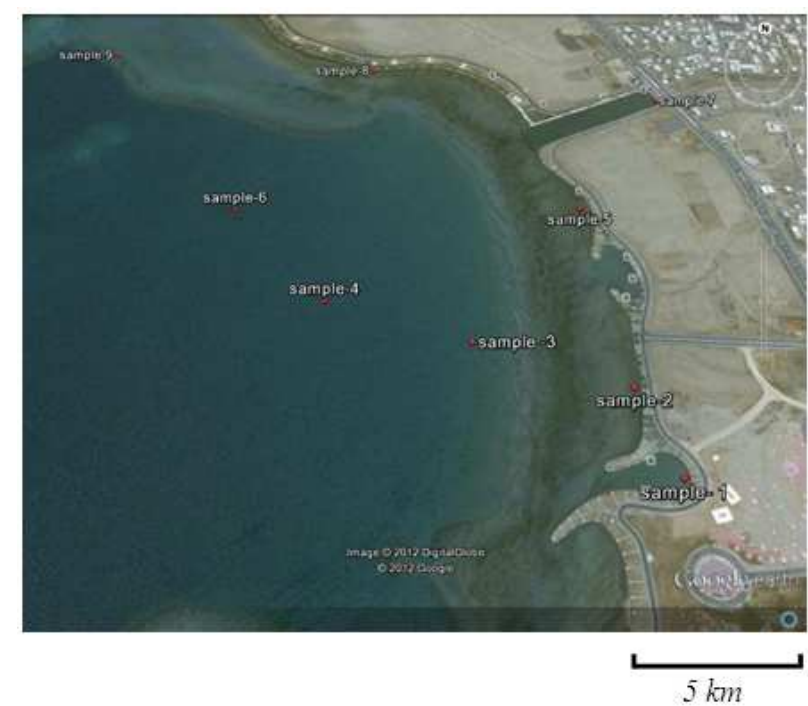

Figure7.Sampling Locations in Al-Qunfudah Beach (South of Saudi Arabia)

\section{Conclusion}

A rapid sensitive method for the determination of TPT, DPT, and MPT in water samples has been developed. The present work uses a novel extraction method based on the use of the dual functionalization of mesoporous silicas with diol and $\mathrm{C}_{18}$ alkane groups for the collection of TPT and its derivatives, DPT and MPT, from water samples. Quantitative recoveries of the target analytes from water samples were achieved using the proposed method. A study of real water samples from different areas of Al-Qunfudah Beach (south of Saudi Arabia) was performed. The limit of detection of $0.5 \mathrm{ngL}^{-1}$ obtained is sufficient for the analysis of real samples since it is lower than the value set by legislation. The results found in this work showed toxic TPT levels. Although the present work uses a new extraction method for water samples, two other significant differences are the following: (1) nanoscavengers can easily deal with large numbers of samples and/or large sample volumes with no risk of hazardous organic solvents and (2) no further intervention was required. All these aspects indicate that the method can be successfully applied to real water samples.

\section{Acknowledgments}

The author thanks the Ministry of Higher Education in Saudi Arabia and Umm Al-Qura University for their financial support. Thanks also go to Mr. Abdulqader Mohammed from Al-Qunfudah University College for his help in collecting the water samples.

\section{References}

[1] H. Shioji, S. Tsunoi, H. Harino, M. Tanaka, "Liquid-phase microextraction of tributyltin and triphenyltin coupled with gas chromatography-tandem mass spectrometry comparison between 4-fluorophenyl and ethyl derivatizations," J. Chromatogr. A, vol. 1048, pp. 81-88, July 2004.

[2] T. Horiguchi, H. Shiraishi, M. Shimizu, M. Morita, "Imposex in sea snails, caused by organotin (tributyltin and triphenyltin) pollution in Japan: Asurvey," Appl. Organomet. Chem., vol. 11, pp. 451-455, May 1997.

[3] J. Holeček, M. Nádvorník, K. Handliř, A. Lyčka, "13C and 119Sn NMR study of some four- and five-coordinate triphenyltin (IV) compounds," J. Organomet. Chem., vol. 241, pp. 177-184, January 1983.

[4] P.E. Gibbs, G.W. Bryan, P.L. Pascoe, G.R. Burt, "The use of the dog-whelk, nucella lapillus, as an indicator of tributyltin (TBT) contamination,'J. Mar. Biol. Ass. UK, vol. 67, pp. 507-523, August 1987.

[5] M. Duft, U. Schulte-Oehlmann, M. Tillmann, B. Markert, J. Oehlmann, "Toxicity of triphenyltin and tributyltin to the freshwater mud snail potamopyrgus antipodarum in a new sediment biotest," Environ Toxicol. Chem., vol. 22, pp. 145-152, January 2003.

[6] D. S. Forsyth, D. Weber, L. Barlow, "The determination of 
organotin compounds in fruit juices using gas chromatography-atomic absorption spectrometry," Appl. Organomet. Chem., vol. 6, pp. 579-585, November 1992.

[7] V. F. Hodge, S. L. Seidel, E. D. Goldberg, "Determination of tin(IV) and organotin compounds in natural waters, coastal sediments and macro algae by atomic absorption spectrometry," Anal. Chem., vol. 51, pp. 1256-1259, July 1979.

[8] J. Szpunar-Łobińska, M. Ceulemans, R. Łobiński, F. C. Adams, "Flow-injection sample preparation for organotin speciation analysis of water by capillary gas chromatography-microwave-induced plasma atomic emission spectrometry," Anal. Chim. Acta., vol. 278, pp. 99-113, June 1993.

[9] G.A. Zachariadis,E. Rosenberg, "Determination of butyland phenyltin compounds in human urine by HS-SPME after derivatization with tetraethylborate and subsequent determination by capillary GC with microwave-induced plasma atomic emission and mass spectrometric detection," Talanta, vol. 78, pp. 570-576, April 2009.

[10] S. Chiron, S. Roy, R. Cottier, R. Jeannot, "Speciation of butyl- and phenyltin compounds in sediments using pressurized liquid extraction and liquid chromatographyinductively coupled plasma mass spectrometry," J. Chromatogr. A, vol. 879, pp. 137-145, May 2000.

[11] C. Bancon-Montigny, G. Lespes, M. Potin-Gautier, "Improved routine speciation of organotin compounds in environmental samples by pulsed flame photometric detection," J. Chromatogr. A, vol. 896, pp. 149-158, October 2000.

[12] S. Díez, L. Ortiz, J.M. Bayona, "Off-line solid-phase extraction procedure for the determination of phenyl and butyltin compounds from aqueous matrices followed by GC-FPD determination," Chromatographia, vol. 52, pp. 657-663, November 2000.

[13] P. Pinel-Raffaitin, P. Rodriguez-Gonzalez, M. Ponthieu, D. Amourouz, I.L. Hecho, L. Mazeas, O.F.X. Donard, M. Potin-Gautier, "Determination of alkylated tin compounds in landfill leachates using isotopically enriched tin species with GC-ICP-MS detection,” J. Anal. At. Spectrom., vol. 22, pp. 258-266, January 2007.

[14] S. Ohhira, H Matsui, "Application of gas chromatographic determination of organotin compounds to basic research on the metabolism of triphenyltin chloride in rats," J. Anal. Toxicol., vol. 16, pp. 375-380, November 1992.

[15] P. Konieczka, B. Sejerøe-Olsen, T. P. J. Linsinger, H. Schimmel, "Determination of tributyltin (TBT) in marine sediment using pressurised liquid extraction-gas chromatography-isotope dilution mass spectrometry (PLEGC-IDMS) with a hexane-tropolone mixture," Anal. Bioanal. Chem., vol. 388, pp. 975-978, June 2007.

[16] A. Wasik, T. Ciesielski, "Determination of organotin compounds in biological samples using accelerated solvent extraction, sodium tetraethylborate ethylation, and multicapillary gas chromatography-flame photometric detection," Anal. Bioanal. Chem., vol. 378, pp. 1357-1363, March 2004.

[17] C. Kalambaheti, P. Wilairat, N. Sakulpaisith, P. Visoottviseth, "Determination of phenyltin compounds by reversed-phase liquid chromatography," Appl. Organomet. Chem., vol. 12, pp. 1-12, January 1998.

[18] J. Darrouzes, M. Bueno, C. Pecheyran, M. Holeman, M. Potin-Gautier, "New approach of solid phase microextraction improving the extraction yield of butyl and phenyltin compounds by combining the effects of pressure and type of agitation," J. Chromatogr. A, vol. 1072, pp. 1927, April 2005.

[19] J. Carpinteiro, I. Rodriquez, R. Cela, "Simultaneous determination of butyltin and phenyltin species in sediments using ultrasound assisted leaching," Fresenius' J. anal. Chem., vol. 370, pp. 872-877, August 2001.

[20] A.G. Howard, N.H. Khdary, "Nanoscavenger based dispersion preconcentration; sub-micron particulate extractants for analyte collection and enrichment," Analyst, vol. 130, pp. 1432-1438, September 2005.

[21] M. M. Algaradah, "The development of nano-sized silicas as analytical tools," Ph.D. Thesis, University of Southampton, Faculty of Natural and Environmental Science, UK November 2010

[22] S.A. Mirji, S.B. Halligudi, D. P. Sawant, N. E. Jacobb, K. R. Patil, A.B. Gaikwadc, S.D. Pradhan, "Adsorption of octadecyltrichlorosilane on mesoporous SBA-15," Appl. Surf. Sci., vol. 252, pp. 4097-4103, April 2006.

[23] H. Y. Huang, C. L. Yang, H. X. Zhang, M. C. Liu, "Preparation and characterization of octyl and octadecylmodified mesoporous SBA-15 silica molecular sieves for adsorption of dimethyl phthalate and diethyl phthalate," Microporous and Mesoporous Mater, vol. 111, pp. 254-259, April 2008.

[24] P. B. Balbuenat, K. E. Gubbins, "Theoretical interpretation of adsorption behavior of simple fluids in slit pores," Langmuir, vol. 9, pp. 1801-1814, March 1993.

[25] A.L. Doadrio, E.M.B. Sousa, J.C. Doadrio, J. Pérez Pariente, I. Izquierdo-Barba, M. Vallet-Regí, "Mesoporous SBA-15 HPLC evaluation for controlled gentamicin drug delivery," J. Controlled Release, vol. 97, pp. 125-132, May 2004. 